\title{
Predicting Cholangitis with Procalcitonin: Procrastinate or Procedure?
}

\author{
Tilak Shah $^{1,2} \cdot$ Alvin Zfass ${ }^{1,2}$ \\ Published online: 8 May 2018 \\ (c) This is a U.S. Government work and not under copyright protection in the US; foreign copyright protection may apply 2018
}

Biliary stasis and increased intraductal pressure are central to the pathogenesis of acute cholangitis (AC). Biliary stasis inhibits the continuous flushing activity of bile and the bacteriostatic effect of bile salts, which help maintain bile sterility. Elevated intraductal pressure favors translocation of bacteria and toxins out of the ducts and into the systemic circulation (i.e., cholangio-venous reflux) [1], which can result in sepsis, shock, and death without timely intervention.

The severity of AC can range from low-grade fevers alone to severe sepsis and shock. Without biliary decompression, the mortality rate of acute cholangitis associated with septic shock approaches $100 \%$. Formerly, surgical emergent biliary decompression was the standard-of-care, but even in expert centers mortality remained unacceptably high at $20-60 \%$ [2]. With the advent of percutaneous biliary drainage (PBD) and subsequently endoscopic retrograde cholangiography (ERC)-guided drainage, the mortality of this condition has strikingly declined to $<5 \%$ [3]. Urgent endoscopic or percutaneous drainage (i.e., within $24 \mathrm{~h}$ ) is now widely accepted as the first-line management for AC associated with signs of septic shock [4].

Given the favorable safety profile of ERC and PTBD, these procedures are now routinely performed in patients with AC who do not have septic shock, although their optimal timing remains a matter of debate, since the benefit of urgent decompression, which has the potential to reduce the risk of progression to septic shock and death, must be weighed against risks, particularly in patients with multiple comorbidities, who may be receiving anticoagulant or antiplatelet medications ("blood thinners") or may be hospitalized in a facility without the necessary expertise in ERC or PTBD. Delaying the procedure in order to optimize

Tilak Shah

Tilak.Shah@va.gov

1 Division of Gastroenterology, 111N, Hunter Holmes McGuire Veterans Affairs Medical Center, 1201 Broad Rock Blvd, Richmond, VA 23224, USA

2 Division of Gastroenterology, Virginia Commonwealth University, Richmond, VA, USA comorbid conditions, appropriately manage blood thinners, and transfer to a center with the necessary expertise thus has the potential to improve outcomes in less severe cases.

Investigators have attempted to develop protocols to triage patients based on clinical and laboratory findings at initial presentation. Among the most frequently cited are the Tokyo consensus guidelines [4] that recommend urgent biliary drainage for moderate-severe AC, but not for mild AC. Unfortunately, retrospective studies assessing the utility of the TG in clinical practice have reported that a number of patients with mild AC subsequently deteriorated [5]. Investigators have proposed several additional variables that predict clinical deterioration (e.g., liver abscess, elevated alanine aminotransferase), but these have not been validated [6]. Thus, the "holy grail," which is a biomarker that accurately identifies which patients with mild or moderate AC are most likely to decompensate despite intravenous fluids and antimicrobial therapy, remains elusive.

Produced by parafollicular cells in the thyroid and neuroendocrine cells of the lung and small intestine, procalcitonin (PCT) is the 116-amino acid peptide precursor of the hormone calcitonin [7]. In health, serum PCT concentrations are below the limit of detection of clinical assays. Systemic inflammation produced by a bacterial infection releases PCT into the bloodstream by the lungs and the intestine, as is the case with other acute phase reactants [7]. Serum PCT concentrations increase with bacterial infections but not viral infections or non-infectious causes of systemic inflammation. Studies conducted in critically ill patients report lower serum PCT concentrations at initial admission in survivors when compared to non-survivors of sepsis $[7,8]$.

In this issue of Digestive Diseases and Sciences, the authors in a study conducted at a single tertiary care hospital in South Korea examined the value of PCT concentrations in the assessment of AC severity and in the prediction of clinical deterioration [9]. At their institution, serum PCT testing is covered by National Health Insurance and is routinely obtained in patients with suspected infectious diseases. The authors retrospectively reviewed serum PCT in 204 patients diagnosed with AC (39 mild AC, 139 moderate AC, and 26 
Fig. 1 Proposed algorithm for use of serum procalcitonin in acute cholangitis, $E R C$ endoscopic retrograde cholangiography; PTC percutaneous transhepatic cholangiography; $P C T$ procalcitonin

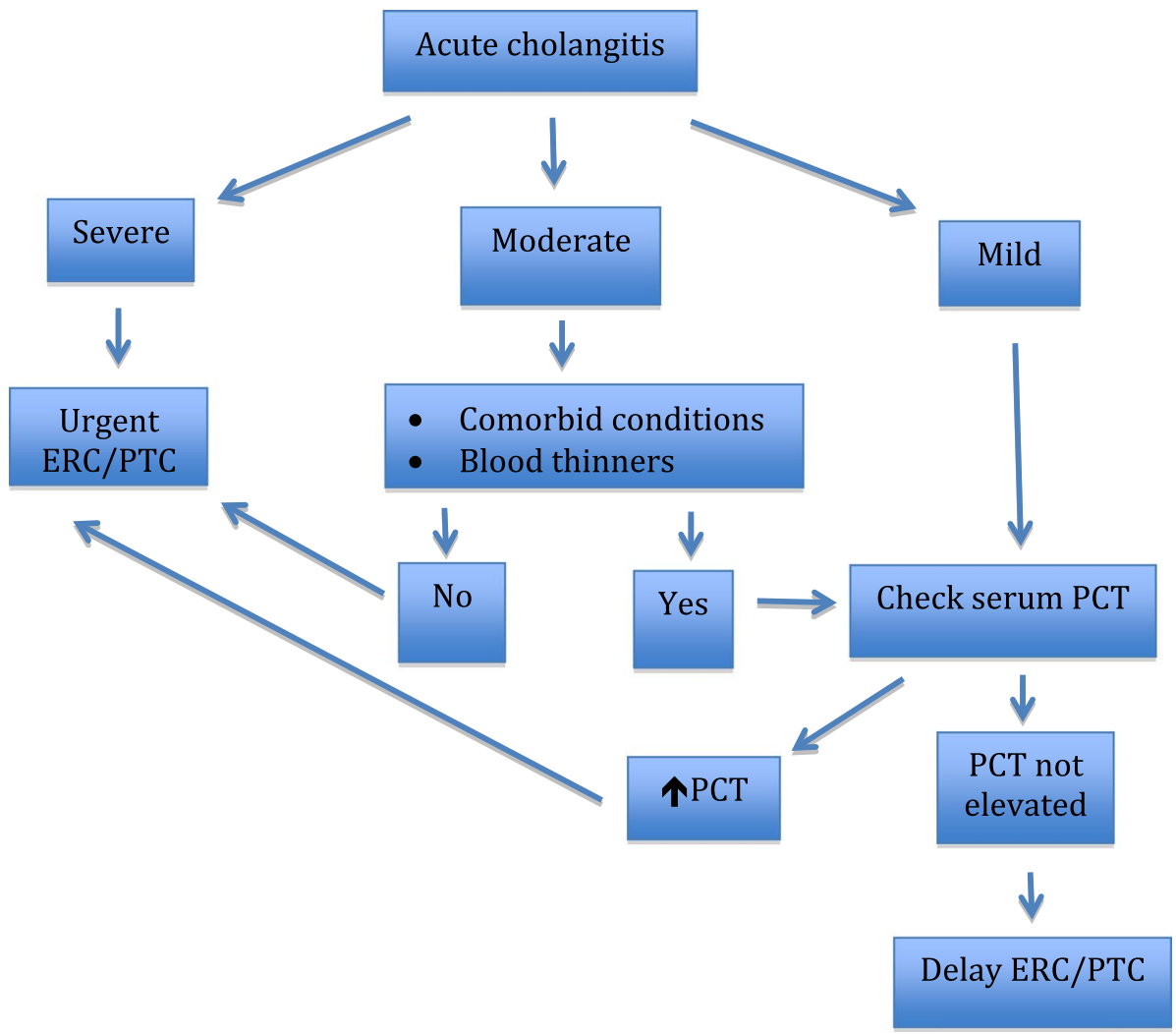

severe AC), 196 of whom were normotensive at initial presentation. Six of these patients subsequently progressed to septic shock before scheduled biliary decompression (1 mild $\mathrm{AC}, 4$ moderate $\mathrm{AC}$, and 1 severe $\mathrm{AC}$ ). In these patients, serum PCT was significantly higher compared to patients who did not decompensate, but white blood cell (WBC) count and C-reactive protein (C-RP) were not statistically different. At a cutoff serum PCT value of $3.77 \mathrm{ng} / \mathrm{mL}$, the investigators calculated sensitivity of $80 \%$ and specificity of $74 \%$ of serum PCT for predicting clinical deterioration in mild and moderate AC. These findings are consistent with those of a prospective study of 213 Japanese patients, which found that serum PCT concentrations were significantly higher among patients with moderate and severe AC compared to mild AC, as defined by the Tokyo guidelines. In patients with severe $\mathrm{AC}$, the area under the receiver operator curve (AUROC) for serum PCT was significantly greater than total WBC count or C-RP [10]. The authors are to be commended for prospectively tracking admission PCT levels over several years in patients with AC. The results do require further validation; however, since this was a retrospective study, without a control arm, limited to a single tertiary care center, with a small number of patients who actually had clinical deterioration.

As is true of most diagnostic tests, serum PCT tests alone are unlikely to replace a clinician's gestalt derived from a synthesis of data obtained from a careful history and physical examination and laboratory and imaging findings. In particular, a low-serum PCT concentration probably should not change a plan for urgent biliary decompression if the clinical picture suggests that the patient has moderate-severe AC.

Where then does serum PCT fit into the management algorithm for $\mathrm{AC}$ ? The test may contribute to determining the timing for urgent biliary decompression in mild AC (Fig. 1). In patients with moderate $\mathrm{AC}$ and other factors that could complicate ERC or PTBD, serum PCT could help assess if the procedure can be delayed with low risk of clinical deterioration (Fig. 1). For instance, take the relatively common scenario of a patient receiving anticoagulant or dual antiplatelet therapy who initially manifests mild or moderate AC due to a bile duct stone. An elevated serum PCT could drive clinical decision making toward pursuing urgent ERC with a stent, followed by repeat ERC at a later date for definitive management (sphincterotomy and stone extraction). On the other hand, if serum PCT is undetectable, a clinician may elect to defer ERC until blood thinners are stopped for a suitable period in order to facilitate definitive therapy to one endoscopic session. Studies that address the utility of serum PCT in such clinical scenarios are eagerly awaited. 


\section{References}

1. Huang T, Bass JA, Williams RD. The significance of biliary pressure in cholangitis. Arch Surg. 1969;98:629-632.

2. Lai EC, Tam PC, Paterson IA, et al. Emergency surgery for severe acute cholangitis. The high-risk patients. Ann Surg. 1990;211:55-59.

3. James PD, Kaplan GG, Myers RP, et al. Decreasing mortality from acute biliary diseases that require endoscopic retrograde cholangiopancreatography: a nationwide cohort study. Clin Gastroenterol Hepatol. 2014;12:1151-1159.

4. Miura F, Okamoto K, Takada T, et al. Tokyo Guidelines 2018: initial management of acute biliary infection and flowchart for acute cholangitis. J Hepatobiliary Pancreat Sci. 2018;25:31-40.

5. Nishino T, Hamano T, Mitsunaga Y, et al. Clinical evaluation of the Tokyo Guidelines 2013 for severity assessment of acute cholangitis. J Hepatobiliary Pancreat Sci. 2014;21:841-849.
6. Salek J, Livote E, Sideridis K, Bank S. Analysis of risk factors predictive of early mortality and urgent ERCP in acute cholangitis. J Clin Gastroenterol. 2009;43:171-175.

7. Arora S, Singh P, Singh PM, Trikha A. Procalcitonin levels in survivors and nonsurvivors of sepsis: systematic review and metaanalysis. Shock. 2015;43:212-221.

8. Prkno A, Wacker C, Brunkhorst FM, Schlattmann P. Procalcitonin-guided therapy in intensive care unit patients with severe sepsis and septic shock-a systematic review and meta-analysis. Crit Care. 2013;17:R291.

9. Lee YS, Cho KB, Park KS. Procalcitonin as a decision-supporting marker of urgent biliary decompression in acute cholangitis. Dig Dis Sci. (Epub ahead of print). https://doi.org/10.1007/s1062 0-018-4963-1.

10. Umefune G, Kogure H, Hamada T, et al. Procalcitonin is a useful biomarker to predict severe acute cholangitis: a single-center prospective study. J Gastroenterol. 2017;52:734-745. 